\title{
Sexual health knowledge in female asylum seekers living in New York City: a single-center, cross-sectional study over one year
}

\author{
Andrew R. Milewski ${ }^{1}$, Mary Cox ${ }^{2}$, Samantha Huynh ${ }^{1}$, Gunisha Kaur ${ }^{1}$, Kane O. Pryor ${ }^{1}$, Sheida Tabaie ${ }^{1} \bullet$ \\ 1 Department of Anesthesiology, Weill Cornell Medicine, New York City, NY, 2 Department of Psychiatry, Weill Cornell Medicine, New York City, NY \\ Keywords: sexual health, asylum seekers, global health \\ https://doi.org/10.29392/001c.24946
}

Journal of Global Health Reports

Vol. 5, 2021

\begin{abstract}
Background
Female asylum seekers remain vulnerable even after resettling in high-income countries. Few studies address sexual health knowledge in this population. This study aims to determine whether female asylum seekers possess less sexual health knowledge than the general female population in New York City (NYC).
\end{abstract}

\section{Methods}

A survey evaluating sexual health knowledge was administered to ten female asylum seekers in NYC and to a general population sample of fifty women in NYC. Two-tailed Student's t-tests were employed to evaluate the statistical significance of the differences in knowledge scores between the two groups, and Fisher's exact tests were used to examine associations between categorical variables.

\section{Results}

On average, female asylum seekers scored 18.8\% lower (95\% confidence interval, $\mathrm{CI}=7.3-30.4, P=0.002)$ on sexual and reproductive health questions, $14.0 \%$ lower $(95 \%$ $\mathrm{CI}=2.8-25.2, P=0.016)$ on contraception questions, $14.3 \%$ lower $(95 \% \mathrm{CI}=3.5-25.1$, $P=0.011$ ) on questions about sexually transmitted diseases (STDs), and $15.1 \%$ lower (95\% $\mathrm{CI}=6.4-23.9, P<0.001)$ overall compared to the control group. Female asylum seekers were, on average, aware of 1.2 fewer $(95 \% \mathrm{CI}=0.1-2.4, P=0.040)$ of the 11 listed contraception methods and of 1.1 fewer (95\% CI=0.3-1.8, $P=0.006$ ) of the five listed STDs.

\section{Conclusions}

Female asylum seekers were found to be less knowledgeable about sexual health than a general population of women in NYC. We additionally highlight a feasible strategy for recruiting participants from a population that is traditionally difficult to access.

Worldwide, more than one billion women-one in every three-have experienced sexual or physical violence in their lifetime. ${ }^{1}$ Many women flee their home countries to seek refuge from violence, conflict, and persecution. The United States grants asylum to thousands of women annually, and more than 100,000 women entered the United States as refugees from 2015 to $2017^{2}$

Evident from the high rates of violence they experience compared to native-born women, female refugees and asylum seekers remain vulnerable even after resettling in highincome countries. ${ }^{3-5}$ Interviews with refugees and other immigrants in Italy revealed that $46.5 \%$ had experienced one or more episodes of violence-at the hands of both citizens and non-citizens-after resettling in the country. ${ }^{6}$ In a study in Belgium and the Netherlands, $58.4 \%$ of the surveyed refugees, asylum seekers, and undocumented migrants personally experienced or knew of a close peer who experienced sexual or gender-based violence since arriving in the European Union. 7
In addition to language barriers, limited access to resources, social isolation, cultural norms, economic disparities, and immigration status, ${ }^{8}$ deficits in sexual health knowledge may contribute to the continued vulnerability experienced by female refugees and asylum seekers after resettlement and may constitute a potentially modifiable risk factor. One third of the refugees, asylum seekers, and undocumented migrants interviewed for a study in the Netherlands and Belgium identified lack of knowledge-including sexual knowledge-as a perceived risk factor linked to sexual victimization; more than half of the respondents felt that preventative strategies should seek to enhance knowledge, including sexual health knowledge. ${ }^{9}$ Unplanned pregnancies occur more often when knowledge of contraceptive methods is lacking, and women who become pregnant unexpectedly are $66 \%$ more likely to experience intimate-partner violence. ${ }^{10}$ Individuals with limited knowledge of sexually transmitted diseases (STDs) are less likely to use condoms consistently and may consequently 
experience higher rates of contracting STDs. ${ }^{11}$

Reflecting the methodological challenges inherent to researching a sensitive topic in small, minority communities, a limited number of studies address sexual health knowledge in refugee populations resettled in high-income countries. ${ }^{12,13}$ Studies that have sought direct comparisons between native-born women and refugees and asylum seekers have primarily focused on evaluating knowledge of the human immunodeficiency virus (HIV) and condoms. Compared to native Australian women, refugee women from West Africa had lower HIV and condom knowledge. ${ }^{14}$ Another study found a deficit in HIV knowledge for young asylum seekers compared to young adult citizens in Finland. ${ }^{15}$ Although they provided no direct comparisons to native-born residents, interview- and survey-based studies of refugees and immigrants in the United States and Europe describe quantifiably low knowledge of HIV/acquired immunodeficiency syndrome, human papillomavirus, contraception, and safer-sex practices. ${ }^{6,16-19}$ Several qualitative reports also suggest that specific populations of refugees who have resettled in the United States, Canada, and Australia possess limited knowledge of sexual health, HIV, and contraceptive methods. ${ }^{20-25}$

By directly comparing their knowledge to that of a control group of female New York City residents, the present study investigates whether female asylum applicants in New York City exhibit knowledge deficits in sexual health, STDs, and contraceptive methods. Because it is located in a high-income country and grants access to a heterogeneous population that includes many foreign-born denizens, New York City constitutes an ideal site for this pilot study.

\section{METHODS}

The Institutional Review Board at Weill Cornell Medicine-the apparatus that ensures the ethical treatment of human research subjects-reviewed and approved this pilot study (protocol \#1806019368). Cisgender, female asylum applicants aged 18 years and older were identified through the Weill Cornell Center for Human Rights (WCCHR) from $1 / 24 / 2019$ to $12 / 13 / 2019$. Asylum seekers are foreign nationals who meet the international definition of refugee and are awaiting adjudication of their petitions for asylum. The WCCHR provides pro bono forensic medical evaluations to asylum applicants and is one of the largest organizations in the United States that offers this service. ${ }^{26}$ To gauge their interest in the study, eligible female asylum applicants were contacted by phone following their forensic medical evaluations with the WCCHR. Those who subsequently enrolled in the study presented to the Weill Cornell Medicine Clinical \& Translational Science Center to give consent and to complete the questionnaire in person. Fifty cisgender, female residents of New York City aged 18 years and older were recruited into the control group by research aides who approached prospective participants in the waiting room of the Primary Care \& Adult Internal Medicine offices at Weill Cornell Medicine. The practice serves a population in and around New York City that is diverse in the socioeconomic classes represented and in the level of education attained by its constituents. Although they were not asked about their citizenship status or country of birth, every woman in the control group had a social security number listed in her medical chart that served as a surrogate for citizenship. No transgender women presented to the WCCHR during the recruitment window; consequently, transgender women were not included in the study.

After providing written, informed consent in their native languages, the study participants completed a written, inperson questionnaire-also in their native languages-that collected demographic data, surveyed attitudes around sexual health practices, and tested knowledge in three subject areas. Specifically, the portion of the questionnaire that evaluated the participants' knowledge contained seven questions concerning general sexual health topics, five questions regarding contraception, and 25 questions about STDs, including HIV (Online Supplementary Document). Participants were also asked to identify the contraceptive methods and STDs that they recognized from lists of 11 and five items, respectively. The questionnaire represents a modest modification of a survey that was previously administered to female university students in Saudi Arabia. ${ }^{27}$

Responses to the questionnaires were de-identified and stored in an institutional REDCap database. All participants answered every item of the questionnaire. The data were exported into R (Version 3.6.3) for statistical analysis. We report the median numbers of correctly answered questions in each subject area and their interquartile ranges in the format: median [ $25^{\text {th }}$ percentile, $75^{\text {th }}$ percentile]. The average knowledge deficit in each subject area was calculated as the difference in the mean number of questions that the control and asylum-applicant groups answered correctly, divided by the number of questions in the category. Odds ratios and average knowledge deficits are reported with 95\% confidence intervals (CI) and significance values. Twotailed Student's t-tests were employed to evaluate the statistical significance of the differences in knowledge scores between the asylum-applicant and control groups, and Fisher's exact tests were used to examine associations between categorical variables. The false discovery rate was controlled at a level of 0.05 using the Benjamini-Hochberg method to account for testing multiple hypotheses. Owing to a small sample size, it was not possible to stratify the asylum applicants' test scores by country of origin, religion, or education level.

The study's funders had no role in designing the study, in analyzing or interpreting the data, or in writing the report. The corresponding author had full access to all of the data in the study and was responsible for the final decision to publish.

\section{RESULTS}

Thirty-five women were eligible for recruitment into the asylum-applicant group: 11 possible participants could not be contacted or moved to another state after their forensic medical evaluation, eight women declined to participate, six women did not appear for their scheduled appointments after expressing interest in the study, and ten of the female asylum applicants were enrolled in the study (Figure 1). The ten asylum applicants fled from eight different countries to escape persecution or abuse: two women hailed from El Salvador; two from Honduras; and one each from the De- 
mocratic Republic of Congo, Ecuador, Guatemala, Mexico, Nigeria, and Togo. Fifty women were enrolled in the control group. The ages of the asylum applicants, $33.8 \pm 10.2$ years (mean \pm standard deviation), were similar to those of the women in the control group, $38.6 \pm 9.9(P=0.200)$. No statistically significant differences between the two groups were found for highest level of education, marital status, age at first childbirth, religious affiliation, or the importance of religion in the respondents' lives.

Compared to the control group, female asylum seekers exhibited a statistically significant knowledge deficit in every area tested. Whereas female asylum applicants answered only $4.5[3.3,5.8]$ out of seven sexual health questions correctly, women in the control group correctly answered 6.0 [5.0,7.0] questions (Figure 2A, "Sexual Health" column), and the asylum applicants' average knowledge deficit was $18.8 \%$ (95\% confidence interval, $\mathrm{CI}=7.3 \%-30.4 \%$, $P=0.002)$. The asylum applicants additionally answered $3.5[3.0,4.0]$ out of five contraception questions correctly and the control group correctly answered 4.0 [4.0,4.0] questions (Figure 2A, "Contraception" column), and the asylum applicants' average knowledge deficit was 14.0\% (95\% $\mathrm{CI}=2.8 \%-25.2 \%, P=0.016)$. In the third area tested-STDs, including HIV-the asylum applicants correctly answered $17.0[14.3,19.0]$ out of 25 questions and women in the control group correctly answered $21.0[18.5,22.0]$, yielding an average deficit of $14.3 \%$ (95\% $\mathrm{CI}=3.5 \%-25.1 \%, P=0.011)$ in the asylum applicants' scores compared to the control group (Figure 2A, "STDs \& HIV" column). After aggregating their responses to all 37 questions, asylum applicants were found to have answered 24.0 [24.0,28.0] questions correctly, compared to the control group who answered 32.0 [27.0,33.0] questions correctly, representing an average knowledge deficit of $15.1 \%$ (95\% CI=6.4\%-23.9\%, $P<0.001$ ) overall (Figure 2A, "All” column).

Female asylum seekers additionally recognized fewer contraceptive methods and STDs compared to women in the control group. Whereas only $7.0[6.3,8.5]$ of the 11 listed contraceptive methods were recognized by the asylum-applicant group, the control group was aware of $9.0[8.0,10.0]$ methods, yielding an average deficit of 1.2 (95\% CI=0.1-2.4, $P=0.040$ ) contraception methods for the asylum applicants compared to the control group (Figure 2B). Similarly, women in the asylum-applicant group recognized only 4.5 $[2.5,5.0]$ STDs, which was less than the $5.0[5.0,5.0]$ STDs recognized by women in the control group. On average, the asylum applicant-group recognized 1.1 fewer (95\% $\mathrm{CI}=0.3-1.8, P=0.006)$ of the five listed STDs than the control group (Figure $2 \mathrm{~B}$ ).

The median score of the asylum-applicant group fell below the $25^{\text {th }}$ percentile score for the control group in every category, and the control group's median score met or surpassed the $75^{\text {th }}$ percentile score for the asylum-applicant group in every category (Figure 2). Only $30 \%$ of the asylum applicants attained scores above $70 \%$ on the questions evaluating sexual health knowledge (37 questions total), far less than the $82 \%$ of women in the control group who surpassed this score. Moreover, overall scores above $85 \%$ were achieved by none of the asylum applicants, and $54 \%$ of women in the control group exceeded this marker. Eight or more contraceptive methods were recognized by only $30 \%$

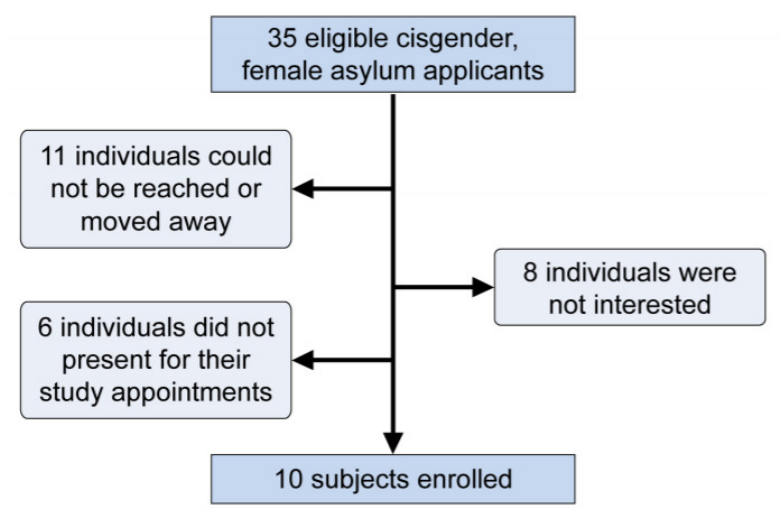

Figure 1. Flow diagram showing the enrollment of cisgender, female asylum applicants for the study

of the asylum applicants, compared to $88 \%$ of the control group. The five listed STDs were recognized by only $50 \%$ of the asylum-applicant group, substantially less than the $92 \%$ of women in the control group who had heard of all the STDs.

One woman in the control group indicated that she recognized only one contraception method and none of the listed STDs, scores that meet Tukey's criteria for far outliers. Considering that she correctly answered 32 out of 37-surpassing the median score in the control group-of the knowledge questions, this participant likely misinterpreted the task of identifying contraception methods and STDs that were known to her.

Among the attitudinal questions, a statistically significant difference was found for the question: "Who are the people you most often talk with about sexual and reproductive health matters?" Participants were asked to choose any of the following options that applied: mother, father, brother/sister, spouse, teacher, religious teacher, friends, other relatives, doctor, pharmacist, nurse, other (Online Supplementary Document). Only 20\% of asylum applicants selected "doctor" from this list, compared to the $82 \%$ of women in the control group who indicated that they most often talk with a doctor about sexual and reproductive health matters, OR 0.06 (95\% CI [0.005,0.358], $P<0.001$ ). Asylum applicants indicated that they instead discussed sexual and reproductive health matters most often with a brother/sister (60\%), friends (50\%), mother (40\%), or spouse (40\%). These proportions did not differ statistically from those for the control group: $22 \%$ discussed these matters with a brother/sister, $58 \%$ with friends, $38 \%$ with their mothers, and $22 \%$ with a spouse.

\section{DISCUSSION}

We found that female asylum seekers living in New York City exhibit significant knowledge deficits in a variety of sexual health topics compared to a control group of women living in New York City. By drawing direct comparisons, we strengthen the evidence supporting previously identified gaps in sexual health knowledge. Our results additionally 
A Sexual Health Knowledge

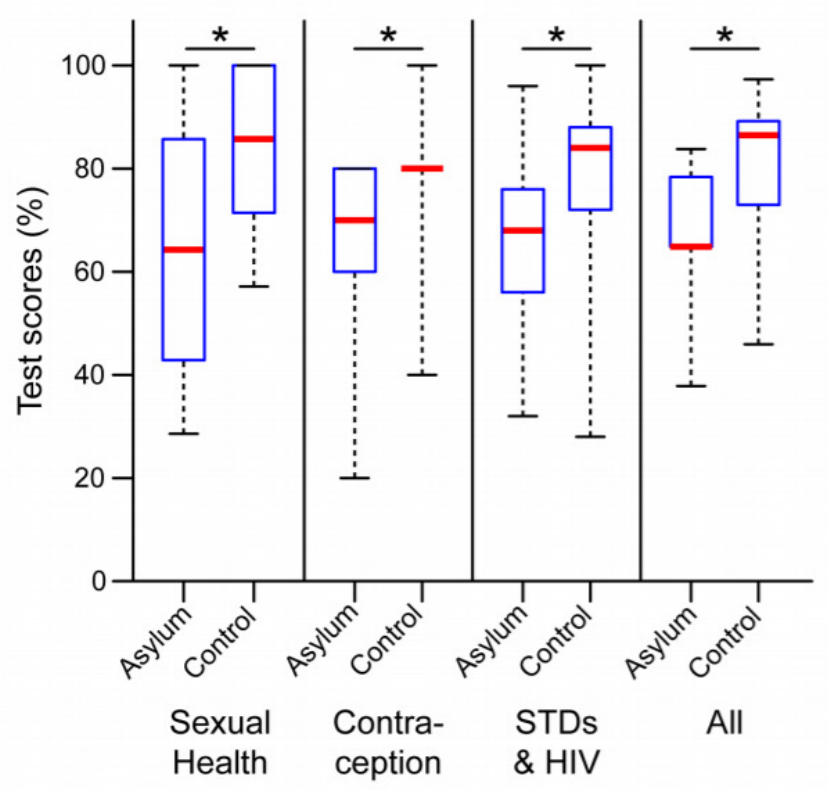

B Methods or STDs Recognized

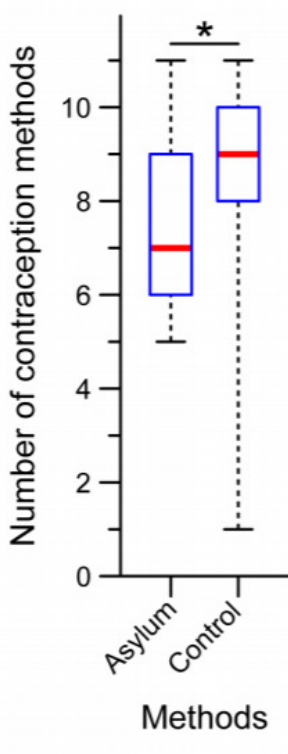

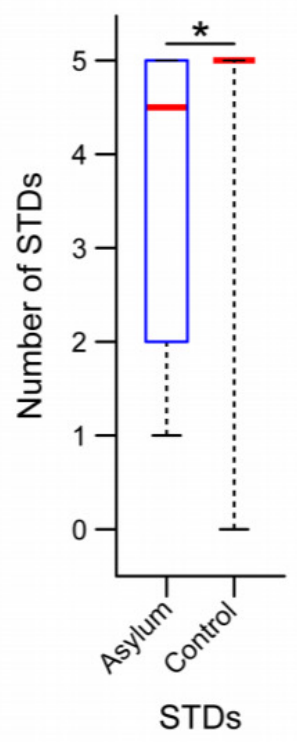

Figure 2. Compared to the control group, asylum-seeking women correctly answered fewer sexual health knowledge questions and recognized fewer contraceptive methods and fewer sexually transmitted diseases

(A) Box-and-whisker diagrams show the median test scores (red lines), together with the interquartile ranges (blue boxes) and overall ranges (black whiskers), for the control and asylum groups in each of the three tested topics-sexual health knowledge, contraception knowledge, and knowledge about sexually transmitted diseases (STDs) including HIV-and in aggregate (All). (B) Box-and-whisker diagrams depict the median numbers (red lines) of contraceptive methods (left) or STDs (right) recognized by the control and asylum groups together with the interquartile ranges (blue boxes) and overall ranges (black whiskers). The lower bound of the whiskers for the control group correspond to the same participant; this individual likely misunderstood that the questionnaire was asking her to indicate the contraception methods and STDs that she recognized. In both panels, asterisks indicate statistically significant differences $(P<0.05)$ between the control and asylum groups.

demonstrate that the deficit in sexual health knowledge exhibited by female asylum seekers resettled in a high-income city like New York extends beyond HIV and condom use. The knowledge gap underscores the multifactorial barriers to obtaining sexual health knowledge, including a disruption in education, separation from family, limited access to health resources and services, religious beliefs, and cultural norms. $8,23,24$ of note, we found that a smaller percentage of women in the asylum-applicant group than in the control group sought to discuss sexual and reproductive health matters with a doctor. This may reflect obstacles-including language barriers, difficulties navigating a new healthcare system, and the competing demands of resettlement-that impact an asylum seeker's desire and ability to access the healthcare system. ${ }^{28,29}$

The present study provides methodological insights for recruiting participants from vulnerable populations. We collaborated with a human rights organization, the Weill Cornell Center for Human Rights (WCCHR), to access a population that is traditionally difficult to reach. Notwithstanding its small sample size, this study demonstrates our ability to successfully connect with female asylum seekers and actively engage them in a study involving a sensitive topic. Partnering with an organization that has substantial connections to the target population therefore constitutes a feasible recruitment strategy for future studies. Although conservative statistical methods ensured that only robust signals were identified, the small number of asylum applicants did, however, preclude the possibility of identifying meaningful trends in their responses to the attitudinal questions and of stratifying the results by the applicants' country of origin or by other demographic factors-including religion and level of education-that may influence baseline sexual health knowledge. Because the WCCHR is one of the most active medical asylum organizations, ${ }^{26}$ achieving the recruitment numbers necessary for subgroup analyses may presently be out of reach for any single center within a reasonable timeframe. Additional recruitment strategies should be considered, including collaborations across multiple organizations providing services to asylum applicants and possibly also partnering with immigration attorneys, asylum officers, or immigration court officials.

There are several additional limitations to this study. By recruiting women for the control group from a waiting room at an internal medicine practice, we may have inadvertently selected individuals with above-average health literacy and, consequently, inflated the knowledge gap between the control and asylum-applicant groups. Future studies might avoid this potential confounder by recruiting participants from community programs or by controlling for participants' engagement with healthcare. The observed effect size could conversely be diminished by unknowingly including foreign-born women in the control group-possibly from countries represented in the asylum-applicant group-who hold green cards or are authorized to work in the United States; the possession of a social security number was used as a surrogate for citizenship and no attempt was made to verify the citizenship status of women in the 
control group. Because we were unable to identify a validated, standardized instrument, we employed a previously published questionnaire with slight modifications to evaluate sexual health knowledge. The limited number of items contained in the questionnaire cannot comprehensively evaluate every part of an individual's sexual health knowledge base.

Despite the aforementioned limitations, the use of conservative statistical methods ensured that only robust signals were identified in our pilot data. These signals point to a relative deficit in sexual health knowledge among female asylum seekers in New York City that provide the foundation for a larger study to corroborate our data. Inadequate sexual health knowledge is a modifiable risk factor with important implications in this population. As the numbers of forcibly displaced people continue to grow worldwide, this issue will become increasingly important. The United Nations High Commissioner for Refugees (UNHCR) estimated that persecution, conflict, violence, human rights violations, and events seriously disturbing public order had forcibly displaced 79.5 million people by the end of 2019 , a number that encompasses 4.2 million individuals awaiting decisions in their asylum claims. ${ }^{30}$ Moreover, two million new asylum applications-accounting for nearly half of the 4.2 million applicants awaiting decisions-were registered in 2019. ${ }^{30}$ Currently home to more than one million applicants awaiting adjudication of their asylum claims, the United States received the largest share of new asylum applications of any country in 2019.30,31

Although investigations of sexual health education in this population are limited, there is strong evidence supporting the positive impact of sexual health curricula on behavior in adolescents and young adults. ${ }^{32,33}$ Delivering a sexual health curriculum to female asylum seekers offers an opportunity to empower a vulnerable population of women by mitigating modifiable risk factors. However, effectively delivering a sexual health curriculum to this population remains challenging. Our data indicate that female asylum seekers in New York City do not routinely seek sexual and reproductive health information from their healthcare providers. To overcome the previously mentioned barriers to accessing the traditional healthcare system, we must consider other avenues for delivering curricula. Through our partnership with the WCCHR, we were able to engage female asylum seekers on sexual and reproductive health topics. Organizations with strong ties to the asylum community, like the WCCHR, may provide a useful alternative to the traditional healthcare system in facilitating the delivery of valuable sexual health knowledge base.

\section{ACKNOWLEDGEMENTS}

The authors gratefully acknowledge Indego Africa-a nonprofit organization that helps transform the lives of women, youth, and refugees in Africa through a market-driven approach to artisan improvement-for its guidance in designing this study. The authors gratefully acknowledge the Weill Cornell Center for Human Rights for granting access to the female asylum seekers who were approached for participation in this study.

\section{FUNDING}

Research reported in this publication was supported by the National Center for Advancing Translational Sciences of the National Institutes of Health under Award Number UL1TR002384. The content is solely the responsibility of the authors and does not necessarily represent the official views of the National Institutes of Health.

\section{AUTHORSHIP CONTRIBUTIONS}

A.M.: formal analysis, visualization, writing - original draft, writing - review \& editing, verified underlying data.

M.C.: data curation, investigation, project administration, resources, verified underlying data.

S.H.: data curation, investigation, project administration, resources, verified underlying data.

G.K.: conceptualization, writing - review \& editing.

K.P.: conceptualization, writing - review \& editing.

S.T.: conceptualization, funding acquisition, project administration, supervision, writing - original draft, writing - review \& editing, verified underlying data.

\section{COMPETING INTEREST}

The authors completed the Unified Competing Interest form at www.icmje.org/coi disclosure.pdf (available upon request from the corresponding author), and declare no conflicts of interest.

\section{CORRESPONDENCE TO:}

Sheida Tabaie, MD

525 East $68^{\text {th }}$ Street

Payson 03-328

New York, NY 10065

Submitted: April 15, 2021 GMT, Accepted: June 10, 2021 GMT 


\section{REFERENCES}

1. United Nations. The World's Women 2015: Trends and Statistics. United Nations, Department of Economic and Social Affairs, Statistics Division; 2015.

2. Mossaad N. Annual Flow Report Refugees and Asylees: 2017. United States Department of Homeland Security, Office of Immigration Statistics; 2019.

3. Hynes M, Lopes Cardozo B. Sexual violence against refugee women. J Womens Health Gend Based Med. 2000;9(8):819-823. doi:10.1089/152460900750020847

4. Freedman J. Sexual and gender-based violence against refugee women: a hidden aspect of the refugee "crisis." Reprod Health Matters. 2016;24(47):18-26. doi:10.1016/i.rhm.2016.05.003

5. Keygnaert I, Vettenburg N, Roelens K, Temmerman M. Sexual health is dead in my body: participatory assessment of sexual health determinants by refugees, asylum seekers and undocumented migrants in Belgium and The Netherlands. BMC Public Health. 2014;14:416. doi:10.1186/1471-2458-1 4-416

6. Napolitano F, Gualdieri L, Santagati G, Angelillo IF. Violence Experience among Immigrants and Refugees: A Cross-Sectional Study in Italy. Biomed Res Int. 2018;2018:7949483. doi:10.1155/2018/794948 $\underline{3}$

7. Keygnaert I, Dias SF, Degomme O, et al. Sexual and gender-based violence in the European asylum and reception sector: a perpetuum mobile? Eur J Public Health. 2015;25(1):90-96. doi:10.1093/eurpub/cku066

8. Runner M, Yoshihama M, Novick S. Intimate Partner Violence in Immigrant and Refugee Communities: Challenges, Promising Practices and Recommendations. Robert Wood Johnson Foundation; 2009.

9. Keygnaert I, Vettenburg N, Temmerman M. Hidden violence is silent rape: sexual and gender-based violence in refugees, asylum seekers and undocumented migrants in Belgium and the Netherlands. Cult Health Sex. 2012;14(5):505-520. do $\mathrm{i}: 10.1080 / 13691058.2012 .671961$

10. Yakubovich AR, Stöckl H, Murray J, et al. Risk and Protective Factors for Intimate Partner Violence Against Women: Systematic Review and Metaanalyses of Prospective-Longitudinal Studies. Am J Public Health. 2018;108(7):e1-e11. doi:10.2105/ajph.2 018.304428
11. Burazeri G, Roshi E, Tavanxhi N. Does knowledge about sexually transmitted infections increase the likelihood of consistent condom use? Prev Med. 2004;39(6):1077-1079. doi:10.1016/i.ypmed.2004.04.0 $\underline{16}$

12. Dean J, Wollin J, Stewart D, Debattista J, Mitchell M. Hidden yet visible: methodological challenges researching sexual health in Sudanese refugee communities. Cult Health Sex. 2012;14(8):911-924. do $\mathrm{i}: 10.1080 / 13691058.2012 .709639$

13. De Schrijver L, Vander Beken T, Krahé B, Keygnaert I. Prevalence of Sexual Violence in Migrants, Applicants for International Protection, and Refugees in Europe: A Critical Interpretive Synthesis of the Evidence. Int J Environ Res Public Health. 2018;15(9):1979. doi:10.3390/ijerph15091979

14. Drummond PD, Mizan A, Wright B. HIV/AIDS knowledge and attitudes among West African immigrant women in Western Australia. Sex Health. 2008;5(3):251-259. doi:10.1071/sh07077

15. Tiittala $P$, Kivelä $P$, Liitsola K, et al. Important Gaps in HIV Knowledge, Attitudes and Practices Among Young Asylum Seekers in Comparison to the General Population. J Immigr Minor Health. 2018;20(6):1415-1423. doi:10.1007/s10903-018-070 $\underline{7-8}$

16. Lazarus JV, Himedan HM, Østergaard LR, Liljestrand J. HIV/AIDS knowledge and condom use among Somali and Sudanese immigrants in Denmark. Scand J Public Health. 2006;34(1):92-99. doi:10.1080/1 $\underline{4034940510032211}$

17. Tompkins M, Smith L, Jones K, Swindells S. HIV education needs among Sudanese immigrants and refugees in the Midwestern United States. AIDS Behav. 2006;10(3):319-323. doi:10.1007/s10461-005-9 060-8

18. Feresu S, Smith L. Knowledge, attitudes, and beliefs about HIV/AIDS of Sudanese and Bantu Somali immigrant women living in Omaha, Nebraska. Open Journal of Preventive Medicine. 2013;3:84-98. do i:10.4236/ojpm.2013.31011

19. Agbemenu K, Aidoo-Frimpong G, Auerbach S, Jaffri A. HIV attitudes and beliefs in U.S.-based African refugee women. Ethn Health. Published online 2020:1-10. doi:10.1080/13557858.2020.1740175

20. McMichael C. Promoting Sexual Health amongst Resettled Youth with Refugee Backgrounds. Refugee Health Research Centre, La Trobe University; 2008. 
21. Ngum Chi Watts MC, Liamputtong P, Carolan M. Contraception knowledge and attitudes: truths and myths among African Australian teenage mothers in Greater Melbourne, Australia. J Clin Nurs.

2014;23(15-16):2131-2141. doi:10.1111/jocn.12335

22. Dhar CP, Kaflay D, Dowshen N, et al. Attitudes and Beliefs Pertaining to Sexual and Reproductive Health Among Unmarried, Female Bhutanese Refugee Youth in Philadelphia. J Adolesc Health. 2017;61(6):791-794. doi:10.1016/j.jadohealth.2017.06.011

23. Metusela C, Ussher J, Perz J, et al. "In My Culture, We Don't Know Anything About That": Sexual and Reproductive Health of Migrant and Refugee Women. Int J Behav Med. 2017;24(6):836-845. doi:10.1007/s12 529-017-9662-3

24. Kingori C, Ice GH, Hassan Q, Elmi A, Perko E. 'If I went to my mom with that information, I'm dead': sexual health knowledge barriers among immigrant and refugee Somali young adults in Ohio. Ethn Health. 2018;23(3):339-352. doi:10.1080/13557858.2016.1263 $\underline{285}$

25. Kaczkowski W, Swartout KM. Exploring gender differences in sexual and reproductive health literacy among young people from refugee backgrounds. Cult Health Sex. 2020;22(4):369-384. doi:10.1080/1369105 $\underline{8.2019 .1601772}$

26. Sharp MB, Milewski AR, Lamneck C, McKenzie K. Evaluating the Impact of Student-run Asylum Clinics in the US from 2016-2018. Health Hum Rights.

2019;21(2):309-323.

27. Farih M, Khan K, Freeth D, Meads C. Protocol study: sexual and reproductive health knowledge, information-seeking behaviour and attitudes among Saudi women: a questionnaire survey of university students. Reprod Health. 2014;11. doi:10.1186/1742-4 755-11-34
28. McMichael C, Gifford S. "It is Good to Know Now...Before it's Too Late”: Promoting Sexual Health Literacy Amongst Resettled Young People With Refugee Backgrounds. Sexuality \& Culture. 2009;13(4):218. doi:10.1007/s12119-009-9055-0

29. McMichael C, Gifford S. Narratives of sexual health risk and protection amongst young people from refugee backgrounds in Melbourne, Australia. Cult Health Sex. 2010;12(3):263-277. doi:10.1080/1369 $\underline{1050903359265}$

30. United Nations High Commissioner for Refugees. Global Trends: Forced Displacement in 2019. UNHCR Global Data Service, Statistics and Demographic Section; 2020.

31. Immigration Court Backlog Tool: Pending Cases and Length of Wait by Nationality, State, Court, and Hearing Location. TRAC Immigration. Accessed June 4, 2020. https://trac.syr.edu/phptools/immigration/co urt backlog/

32. Drummond PD, Mizan A, Brocx K, Wright B. Using peer education to increase sexual health knowledge among West African refugees in Western Australia. Health Care Women Int. 2011;32(3):190-205. doi:10.10 $\underline{80 / 07399332.2010 .529215}$

33. Kirby DB, Laris BA, Rolleri LA. Sex and HIV education programs: their impact on sexual behaviors of young people throughout the world. J Adolesc Health. 2007;40(3):206-217. doi:10.1016/j.jadohealt h.2006.11.143 


\section{SUPPLEMENTARY MATERIALS}

\section{Online Supplement Document}

Download: https://www.joghr.org/article/24946-sexual-health-knowledge-in-female-asylum-seekers-living-in-newyork-city-a-single-center-cross-sectional-study-over-one-year/attachment/63441.pdf 